\title{
ALTERAÇÕES FUNCIONAIS RESPIRATÓRIAS NA DOENÇA DE PARKINSON
}

\begin{abstract}
Henrique da Conceição Costa*, Bárbara Silva dos Santos*, Paula Silva Oliveira*, loná da Costa Barreto Nascimento**, Ana Caline Nóbrega***, Fernanda Warken Rosa Camelier****, Grupo de Pesquisa Estudo e Pesquisa da Funcionalidade/UNEB *****, Grupo de Pesquisa DINEP - Divisão de Neurologia e Epidemiologia/UFBA ${ }^{* * * * * *}$
\end{abstract}

Autor correspondente: Fernanda Warken Rosa Camelier - fcamelier@uneb.br

* Graduando (a) em Fisioterapia, Departamento de Ciências da Vida, Universidade do Estado da Bahia.

** Fisioterapeuta, Departamento de Ciências da Vida, Universidade do Estado da Bahia.

*** Fonoaudiológa, PhD, Professora Adjunta, Divisão de Neurologia e Epidemiologia do Departamento de Neurociências e Saúde Mental, Universidade Federal da Bahia.

**** Fisioterapeuta, PhD, Professora Titular, Departamento de Ciências da Vida, Universidade do Estado da Bahia.

\section{Resumo}

Introdução: Caracterizada como uma patologia crônica, neurodegenerativa e progressiva, a Doença de Parkinson (DP) está relacionada a disfunções monoaminérgicas múltiplas, incluindo déficits dos sistemas dopaminérgicos, colinérgicos, serotoninérgicos e noradrenérgicos. Sintomas motores clássicos como a rigidez, bradicinesia, tremor em repouso e instabilidade postural são presentes nesta condição. Derivada desta tétrade motora, as alterações posturais, a rigidez e a bradicinesia levam à redução da força muscular respiratória, modificam a biomecânica da caixa torácica causando déficits na expansão pulmonar, podendo ainda, repercutir nos volumes e capacidades pulmonares. Objetivo: Sistematizar o conhecimento acerca das alterações funcionais respiratórias presentes na doença de Parkinson. Metodologia: Trata-se de um estudo de Revisão de Literatura. As bases de dados pesquisadas foram Bireme, Scielo e PubMed, e foram incluídos artigos originais, publicados a partir do ano de 2000 , que avaliaram a função pulmonar com medidas da manobra de capacidade vital forçada (espirometria), pico de fluxo de tosse, força muscular respiratória e a mobilidade torácica. Foram excluídos artigos que avaliaram a função pulmonar com outros desfechos e aqueles que não foram disponibilizados na íntegra. Resultados: Foram encontrados 45 artigos, e após a aplicação dos critérios de inclusão e exclusão, foram selecionados 16 estudos. Considerações Finais: Esta revisão de literatura sugere que pessoas com Parkinson apresentam redução da mobilidade torácica, do pico de fluxo de tosse, da força muscular respiratória e de volumes e capacidades pulmonares, implicando no comprometimento da funcionalidade destes indivíduos.

Palavras-chave: Doença de Parkinson; Testes de Função Respiratória; Avaliação. 


\title{
RESPIRATORY DYSFUNCTIONS IN PARKINSON'S DISEASE \\ - state of art
}

\begin{abstract}
Introduction: Parkinson's Disease (PD) is a chronic progressive neurodegenerative pathology related to a multiple monoaminergic dysfunctions, including dopaminergic, cholinergic, serotoninergic and noradrenergic systems deficits. Classic motor system deficits like stiffness, bradikynesia, postural instability and tremors are present in such conditions. The motor system deficits lead to a reduction in respiratory muscle function and the lung mechanics and respiratory function are then modified. Objective: To summarize the PD impact on respiratory function knowledge through a systematized literature review. Methods: This is a literature review study. Bibliographic search was conducted in Bireme, Scielo and PubMed databases. Original papers published from January first, 2000 to June first 2016 were selected. Papers with pulmonary function with measurement of forced vital capacity, cough peak flow, respiratory muscle strength and thoracic mobility were included. Papers with other outcomes and those whose authors had not acess to full text versions were excluded. Results: Forty-five papers were initially selected, being 29 excluded. At the end, sixteen papers entered in the study, and two of them evaluated thoracic mobility, 11 evaluated cogh peak flow and forced vital capacity, and four evaluated respiratory muscle strength. Final Considerations: This literature review suggests that subjects with PD have a reduction of thoracic mobility, cough peak flow, respiratory muscle strength and lung volumes and capacity, leading to a compromised functionality of this subjects.
\end{abstract}

Kepwords: Parkinson disease; Respiratory Function Tests; Evaluation.

\section{INTRODUÇÃO}

A Doença de Parkinson (DP) é uma patologia neurodegenerativa crônica e progressiva, relacionada a disfunções monoaminérgicas múltiplas, incluindo déficits dos sistemas dopaminérgicos, colinérgicos, serotoninérgicos e noradrenérgicos. É descrita com clareza a existência de uma perda neuronal progressiva no grupo de células ventro-laterais, da parte compacta da substância negra do mesencéfalo. (1) Em situações ideais, para a realização do movimento voluntário, há um aumento na atividade de neurônios talâmicos e do córtex cerebral, facilitando sua execução. A diminuição na concentração de dopamina altera o funcionamento do núcleo estriado tornando-o excessivamente ativo, dificultando o controle do movimento pela pessoa com DP. ${ }^{(2-4)}$

Os principais sintomas motores formam uma tétrade representada pela rigidez muscular, bradicinesia, tremor de repouso e instabilidade postural. ${ }^{(5)}$ Outros sintomas de cunho não motor como distúrbios cognitivos, do sono e depressão também podem estar presentes. ${ }^{(6,7)} \mathrm{Na}$ DP as alterações posturais, a rigidez e a bradicinesia modificam a biomecânica da caixa torácica, causando déficits na expansão pulmonar que podem determinar impactos na função respiratória. ${ }^{(8)}$ 
Embora as disfunções respiratórias na DP sejam multicausais, as alterações motoras têm sido relacionadas as mesmas, de modo a comprometer a ventilação alveolar e qualidade das trocas gasosas em parkinsonianos. (9) Estas alterações envolvem fatores como a redução da força e da mobilidade torácica, anormalidades posturais, perturbações na ativação e coordenação muscular, que consequentemente geram limitações na capacidade ventilatória, aumentando a resistência ao fluxo aéreo, restringindo a expansão torácica. ${ }^{(8,9)}$

A postura em flexão e a rigidez articular comprometem a mobilidade torácica, prejudicando a função pulmonar e consequentemente a complacência, o que repercute no aumento dos distúrbios ventilatórios que podem ser obstrutivos e/ou restritivos. ${ }^{(6,9)}$ Ainda como consequência da rigidez muscular e bradicinesia, encontram-se presentes a redução da pressão inspiratória e expiratória máxima, que refletem a força muscular. ${ }^{(8)}$ A DP é uma das patologias mais frequentes em pessoas idosas, e as alterações no sistema respiratório se associam ao comprometimento próprio do envelhecimento tais como, redução da elasticidade, dilatação alveolar, diminuição de estímulos neurais para músculos da respiração e alterações de volumes, capacidades e fluxos respiratórios.(7)

Métodos de avaliação funcional tais como a cirtometria torácica, a manovacuometria, a medida do pico de fluxo de tosse e a espirometria podem ser utilizados para avaliar a mobilidade torácica, a força muscular respiratória, a tosse e os volumes e capacidades pulmonares, respectivamente. Identificar a presença de alterações respiratórias nessa população auxilia a prevenção e no direcionamento terapêutico de diversas condições clinicas associadas. O objetivo do estudo foi sistematizar o conhecimento acerca das alterações funcionais respiratórias presentes na doença de Parkinson.

\section{MÉTODOS}

Trata-se de um estudo de revisão de literatura. As bases de dados utilizadas na pesquisa foram PubMed, SciELO, e Bireme utilizando as palavras-chave: "cirtometria", "doença de Parkinson", "espirometria", "força muscular respiratória", "função pulmonar", "métodos de avaliação", "mobilidade torácica", "pico de fluxo expiratório", "pico de fluxo de tosse", "pressão expiratória máxima" e "pressão inspiratória máxima", acrescidas dos operadores booleanos "AND" e "OR" nos idiomas inglês e português. Foram incluídos artigos científicos originais que avaliaram a função respiratória em pacientes com doença de Parkinson publicados a partir do ano 2000, publicados nos idiomas português e inglês. Artigos não disponibilizados na íntegra foram excluídos. Os desfechos avaliados foram medidas da manobra de capacidade vital forçada (espirometria), pico de fluxo de tosse, força muscular respiratória e a mobilidade torácica.

\section{RESULTADOS}

A busca de artigos nas bases de dados resultou em 45 artigos. Destes, 15 artigos foram encontrados na Bireme, sendo que quatro foram incluídos; dos três artigos selecionados na SciELO, apenas um estudo foi incluído; e dos 27 estudos encontrados no Pubmed, 11 atenderam aos critérios de seleção. Ao final da análise pelos critérios de elegibilidade foram incluídos 16 estudos para a revisão, sendo 12 publicações estrangeiras. A tabela 1 apresenta uma síntese das características metodológicas e os principais desfechos clínicos avaliados nos estudos exibidos por ano de publicação. A mobilidade torácica foi avaliada por dois estudos. Onze estudaram o pico de fluxo de tosse, quatro a força muscular respiratória e 11 dissertaram sobre a capacidade vital forçada. 
Tabela 1 - Característica dos artigos incluídos no estudo sobre a sistematização das alterações funcionais respiratórias na Doença de Parkinson, apresentados em decrescente por ano de publicação

(continua)

\begin{tabular}{|c|c|c|c|c|}
\hline $\begin{array}{l}\text { AUTOR(ES)/ } \\
\text { ANO/ PAÍS }\end{array}$ & $\begin{array}{l}\text { TIPO DE } \\
\text { ESTUDO }\end{array}$ & $\begin{array}{l}\text { CARACTERÍSTICAS } \\
\text { DA POPULAÇÃO }\end{array}$ & DESFECHO(s) & RESULTADO(s) ENCONTRADO(s) \\
\hline $\begin{array}{l}\text { Owolabi et al., } \\
\text { 2016. Nigéria }\end{array}$ & $\begin{array}{l}\text { Caso- } \\
\text { Controle }\end{array}$ & $\begin{array}{l}78 \text { indivíduos } \\
\text { (60 homens) }\end{array}$ & $\begin{array}{l}\text { Capacidade vital } \\
\text { forçada, pico de } \\
\text { fluxo expiratório }\end{array}$ & $\begin{array}{l}\text { A CV foi significantemente menor } \\
\text { em indivíduos com Doença de } \\
\text { Parkinson em comparação ao } \\
\text { controle ( } \mathrm{p}<\mathrm{O}, \mathrm{O} O \mathrm{O}) \text {. A média } \\
\text { dos valores de pico de fluxo } \\
\text { expiratório nos indivíduos com } \\
\text { DP foi significantemente menor, } \\
\text { alcançando a média de } 45,58 \% \\
\text { do previsto, com } \mathrm{p}<\mathrm{O}, \mathrm{OOO} \text { em } \\
\text { comparação ao controle. }\end{array}$ \\
\hline $\begin{array}{l}\text { Silverman et } \\
\text { al., 2016. EUA }\end{array}$ & Transversal & $\begin{array}{l}68 \text { pessoas } \\
\text { (55 homens) }\end{array}$ & $\begin{array}{l}\text { Pico de fluxo de } \\
\text { tosse }\end{array}$ & $\begin{array}{l}\text { Os três subgrupos de indivíduos } \\
\text { com DP (<60 anos; 60-69 anos; } \\
\text { >7O anos) apresentaram valores } \\
\text { PFT abaixo da normalidade. }\end{array}$ \\
\hline $\begin{array}{c}\text { Ramos et al., } \\
\text { 2014. Brasil }\end{array}$ & Transversal & $\begin{array}{c}10 \text { indivíduos (3 } \\
\text { homens) }\end{array}$ & $\begin{array}{l}\text { Mobilidade torácica } \\
\text { e pico de fluxo de } \\
\text { tosse }\end{array}$ & $\begin{array}{c}\text { Mobilidade torácica reduzida } \\
\text { em } 70 \% \text { dos estudados e } 90 \% \\
\text { apresentaram baixos valores PFT. }\end{array}$ \\
\hline $\begin{array}{l}\text { Wang et al., } \\
\text { 2014. China }\end{array}$ & $\begin{array}{l}\text { Caso- } \\
\text { controle }\end{array}$ & $\begin{array}{l}30 \text { indivíduos } \\
\text { com DP (16 } \\
\text { homens); } 20 \\
\text { indivíduos } \\
\text { hígidos (10 } \\
\text { homens) }\end{array}$ & $\begin{array}{l}\text { Força muscular } \\
\text { respiratória, } \\
\text { capacidade vital } \\
\text { forçada, pico de } \\
\text { fluxo expiratório }\end{array}$ & 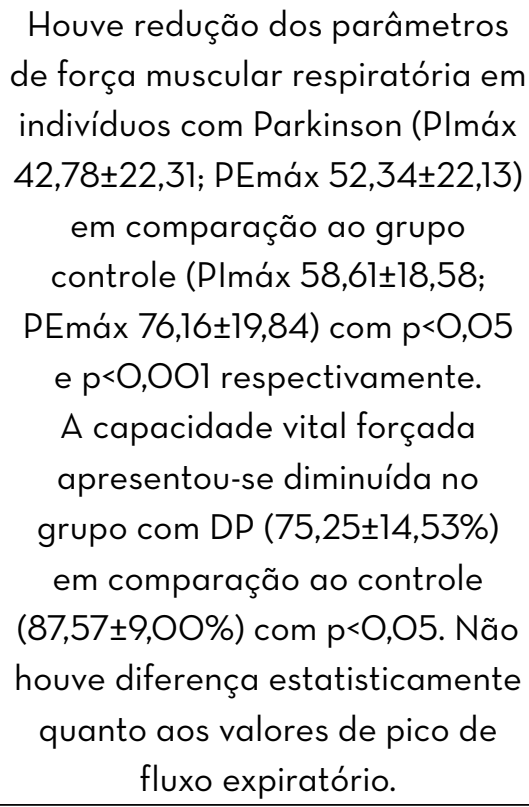 \\
\hline $\begin{array}{c}\text { Bonjorni et al., } \\
\text { 2012. Brasil }\end{array}$ & $\begin{array}{l}\text { Caso- } \\
\text { controle }\end{array}$ & $\begin{array}{l}10 \text { indivíduos } \\
\text { com Parkinson, } \\
\text { (8 homens). } \\
\text { Grupo controle } \\
\text { composto por } \\
15 \text { sujeitos, (12 } \\
\text { homens) }\end{array}$ & $\begin{array}{l}\text { Capacidade } \\
\text { vital forçada, e } \\
\text { força muscular } \\
\text { respiratória }\end{array}$ & $\begin{array}{l}\text { Todos os casos apresentaram } \\
\text { distúrbio ventilatório obstrutivo. } \\
\text { O grupo doença de Parkinson } \\
\text { apresentou redução da PImáx } \\
(4 \mathrm{O} \pm 43,7) \text { e PEmáx }(77 \pm 43,2) \\
\text { comparado ao grupo controle } \\
\text { Plmáx }(77 \pm 13,3) \text { e PEmáx }(105,7 \\
\pm 17,8) \text {, com p, respectivamente, } \\
\text { O,O4 e } 0,03 \text {. }\end{array}$ \\
\hline
\end{tabular}


Tabela 1 - Característica dos artigos incluídos no estudo sobre a sistematização das alterações funcionais respiratórias na Doença de Parkinson, apresentados em decrescente por ano de publicação

(continuação)

\begin{tabular}{|c|c|c|c|c|}
\hline $\begin{array}{c}\text { Guedes et al., } \\
\text { 2012. Brasil }\end{array}$ & $\begin{array}{l}\text { Caso- } \\
\text { controle }\end{array}$ & $\begin{array}{l}26 \text { indivíduos } \\
\text { com Parkinson } \\
\text { (16 homens). } \\
\text { Grupo controle } \\
\text { composto por } \\
26 \text { indivíduos ( } 16 \\
\text { homens) }\end{array}$ & $\begin{array}{l}\text { Força muscular } \\
\text { respiratória (em } \\
\text { estados on e off da } \\
\text { medicação) }\end{array}$ & $\begin{array}{c}\text { A PImáx e a PEmáx } \\
\text { apresentaram-se reduzidas no } \\
\text { grupo com Parkinson, quando } \\
\text { comparou-se com os indivíduos } \\
\text { saudáveis, não havendo diferença } \\
\text { estatisticamente significante } \\
\text { entre os períodos on e off. }\end{array}$ \\
\hline $\begin{array}{l}\text { Seccombe } \\
\text { et al., } 2011 . \\
\text { Austrália }\end{array}$ & Transversal & $\begin{array}{c}19 \text { indivíduos (17 } \\
\text { homens) }\end{array}$ & $\begin{array}{l}\text { Capacidade } \\
\text { vital forçada e } \\
\text { força muscular } \\
\text { respiratória, pico de } \\
\text { fluxo expiratório }\end{array}$ & $\begin{array}{l}\text { 15,8\% apresentaram alteração } \\
\text { na função pulmonar. Um (5,3\%) } \\
\text { paciente com limitação ao fluxo } \\
\text { aéreo leve e dois (10,5\%) foram } \\
\text { classificados como restritivos. } \\
\text { Plmáx abaixo do limite inferior } \\
\text { de normalidade em 68\% dos } \\
\text { indivíduos estudados; PEmáx } \\
\text { abaixo desse limite em 79\% da } \\
\text { amostra. Os valores de pico de } \\
\text { fluxo expiratório apresentaram-se } \\
\text { dentro da faixa de normalidade. }\end{array}$ \\
\hline $\begin{array}{c}\text { Shaheen et al., } \\
\text { 2009. Egito }\end{array}$ & $\begin{array}{l}\text { Caso- } \\
\text { Controle }\end{array}$ & $\begin{array}{c}30 \text { indivíduos } \\
\text { com DP ( } 28 \\
\text { homens). } 30 \\
\text { indivíduos no } \\
\text { grupo controle } \\
\text { (28 homens) }\end{array}$ & $\begin{array}{c}\text { Capacidade vital } \\
\text { forçada }\end{array}$ & $\begin{array}{l}\text { Em comparação ao controle, os } \\
\text { indivíduos com DP apresentaram } \\
\text { redução do parâmetro CVF, com } \\
\qquad p=0,000 \text {. }\end{array}$ \\
\hline $\begin{array}{c}\text { Lim et al., } \\
\text { 2007. Nova } \\
\text { Zelândia }\end{array}$ & Transversal & $\begin{array}{l}10 \text { indivíduos } \\
\text { com (6 homens) }\end{array}$ & $\begin{array}{l}\text { Capacidade vital } \\
\text { forçada (em estados } \\
\text { "on" e "off" da } \\
\text { medicação, pico de } \\
\text { fluxo expiratório }\end{array}$ & $\begin{array}{c}\text { Testes de função pulmonar } \\
\text { mostraram melhorias } \\
\text { significativas em estado "off' da } \\
\text { medicação na CVF (O,15 L, p = } \\
\text { O,OO2). Não houve diferença } \\
\text { estatisticamente entre os } \\
\text { períodos on e off. }\end{array}$ \\
\hline $\begin{array}{c}\text { Pal et al., } 2007 . \\
\text { Índia }\end{array}$ & Transversal & $\begin{array}{c}53 \text { indivíduos } \\
\text { com DP (38 } \\
\text { homens) }\end{array}$ & $\begin{array}{l}\text { Capacidade vital } \\
\text { forçada, VEF }(\mathrm{em} \\
\text { estados "on" e "off" } \\
\text { da medicação, pico } \\
\text { de fluxo expiratório }\end{array}$ & 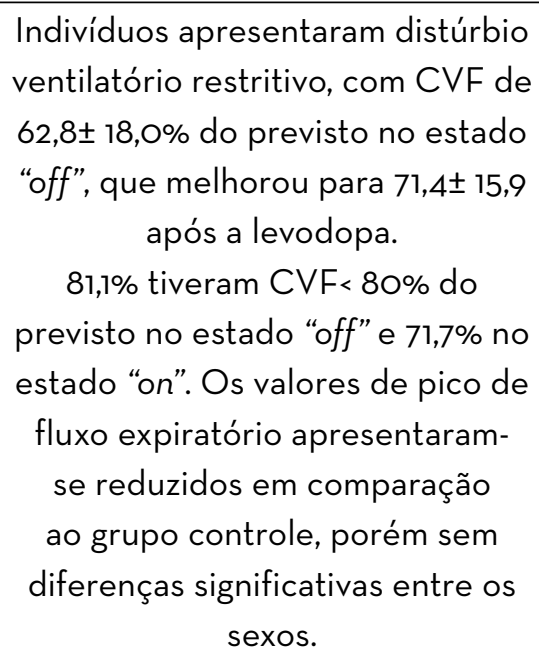 \\
\hline
\end{tabular}


Tabela 1 - Característica dos artigos incluídos no estudo sobre a sistematização das alterações funcionais respiratórias na Doença de Parkinson, apresentados em decrescente por ano de publicação

\begin{tabular}{|c|c|c|c|c|}
\hline $\begin{array}{c}\text { Sathyaprabha } \\
\text { et al., } 2005 . \\
\text { Índia }\end{array}$ & $\begin{array}{l}\text { Caso- } \\
\text { controle }\end{array}$ & $\begin{array}{c}35 \text { indivíduos } \\
\text { com DP. Grupo } \\
\text { controle com } 54 \\
\text { sujeitos. }\end{array}$ & $\begin{array}{l}\text { Capacidade } \\
\text { vital forçada (em } \\
\text { estados "on" e "off" } \\
\text { da medicação e } \\
\text { força muscular } \\
\text { respiratória, pico de } \\
\text { fluxo expiratório }\end{array}$ & 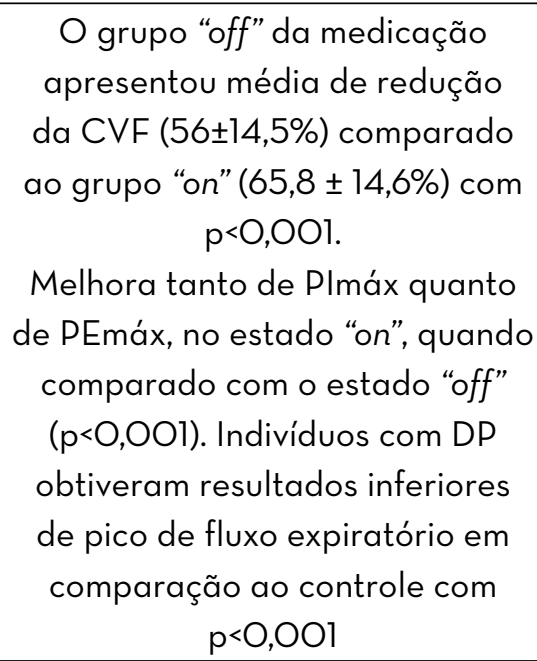 \\
\hline $\begin{array}{l}\text { Ebihara et al., } \\
\text { 2003. Japão }\end{array}$ & $\begin{array}{l}\text { Caso- } \\
\text { Controle }\end{array}$ & $\begin{array}{c}25 \text { mulheres } \\
\text { com DP. Grupo } \\
\text { controle } \\
\text { constituído por } \\
16 \text { participantes }\end{array}$ & $\begin{array}{l}\text { Capacidade vital } \\
\text { forçada e pico de } \\
\text { fluxo de tosse }\end{array}$ & $\begin{array}{c}\text { CVF reduzida no estágio } \\
\text { avançado }(75,6 \pm 20,0) \text {, } \\
\text { comparado ao estágio inicial } \\
(82,1 \pm 9,0 \text { ) e ao controle }(87,1 \pm 12,1) \text {. } \\
\text { O PFT tanto em pacientes com DP } \\
\text { em fase inicial }(230 \pm 74 \mathrm{~L} / \mathrm{min} ; \mathrm{p}< \\
\text { o,005) e avançada ( } 186 \pm 60 \mathrm{~L} / \mathrm{min} \text {; } \\
\text { p<0,0001) foram significativamente } \\
\text { mais fraca do que a intensidade da } \\
\text { do grupo controle }(316 \pm 70 \mathrm{~L} / \mathrm{min}) \text {. }\end{array}$ \\
\hline $\begin{array}{c}\text { Cardoso e } \\
\text { Pereira, } 2002 . \\
\text { Brasil }\end{array}$ & $\begin{array}{l}\text { Caso- } \\
\text { controle }\end{array}$ & $\begin{array}{l}40 \text { indivíduos } \\
\text { com DP ( } 21 \\
\text { homens), } \\
\text { Grupo controle } \\
\text { constituído por } \\
40 \text { sujeitos }\end{array}$ & $\begin{array}{l}\text { Força muscular } \\
\text { respiratória, } \\
\text { capacidade } \\
\text { vital forçada e } \\
\text { mobilidade torácica. }\end{array}$ & $\begin{array}{l}\text { Plmáx de } 33,5 \pm 12,7 \text { nos } \\
\text { Parkinsonianos e } 37 \pm 12,2 \mathrm{cmH}_{2} \mathrm{O} \\
\text { no grupo controle e PEmáx, } \\
\text { respectivamente, } 36,3 \pm 17,8 \text { e } \\
43,1 \pm 16,6 \mathrm{cmH}_{2} \mathrm{O} \text {. Sujeitos com DP } \\
\text { apresentaram redução da CVF } \\
\text { em relação aos valores teóricos, } \\
\text { de } 66,8 \pm 20,3 \% \text { nos Parkinsonianos } \\
\text { e } 82,3 \pm 15,7 \% \text { nos controle com } \\
\text { p=0,00001. De forma semelhante, } \\
\text { a mobilidade torácica foi } \\
\text { significativamente menor no grupo } \\
\text { Parkinsoniano ( } \mathrm{p}=0,00001 \text { ). } \\
\text { Houve } 55 \% \text { de casos de restrição } \\
\text { em parkinsonianos. }\end{array}$ \\
\hline $\begin{array}{l}\text { Pandis et al., } \\
\text { 2002. Itália }\end{array}$ & Transversal & $\begin{array}{l}12 \text { participantes } \\
\text { (5 homens) }\end{array}$ & $\begin{array}{l}\text { Capacidade vital } \\
\text { forçada (em estados } \\
\text { "on" e "off" da } \\
\text { medicação, pico de } \\
\text { fluxo expiratório }\end{array}$ & $\begin{array}{c}\text { Observou-se CVF reduzida em } \\
\text { relação aos valores previstos em } \\
\text { todos os estágios, com melhora } \\
\text { na fase "on" ( } p<0,005) \text {. Houve } \\
\text { melhora nos valores de pico de } \\
\text { fluxo expiratório no estado on da } \\
\text { medicação. }\end{array}$ \\
\hline
\end{tabular}


Tabela 1 - Característica dos artigos incluídos no estudo sobre a sistematização das alterações funcionais respiratórias na Doença de Parkinson, apresentados em decrescente por ano de publicação

\begin{tabular}{|c|c|c|c|c|}
\hline $\begin{array}{l}\text { Herer et al., } \\
\text { 2001. França }\end{array}$ & Transversal & $\begin{array}{l}21 \text { indivíduo com } \\
\text { DP (14 homens) }\end{array}$ & $\begin{array}{l}\text { Capacidade vital } \\
\text { forçada (em estados } \\
\text { "on" e "off" da } \\
\text { medicação, pico de } \\
\text { fluxo expiratório }\end{array}$ & $\begin{array}{c}\text { Não houve diferença } \\
\text { estatisticamente significante } \\
(p=0,08) \text { nos valores da CVF após } \\
\text { a utilização da medicação em } \\
\text { indivíduos com Parkinson, com } \\
\text { e sem obstrução das vias aéreas } \\
\text { superiores. Não houve diferença } \\
\text { estatisticamente significante } \\
\text { dos valores de pico de fluxo } \\
\text { expiratório após a utilização da } \\
\text { medicação. }\end{array}$ \\
\hline $\begin{array}{l}\text { Polatli et al., } \\
\text { 2001. Turquia }\end{array}$ & $\begin{array}{l}\text { Caso- } \\
\text { Controle }\end{array}$ & $\begin{array}{l}21 \text { indivíduos } \\
\text { com DP (1O } \\
\text { homens) e } \\
21 \text { indivíduos } \\
\text { controle }\end{array}$ & $\begin{array}{l}\text { Capacidade vital } \\
\text { forçada, pico de } \\
\text { fluxo expiratório }\end{array}$ & $\begin{array}{l}\text { Não houve diferença estatística } \\
\text { da CVF em comparação ao } \\
\text { controle, sendo evidenciado nos } \\
\text { parkinsonianos CVF }(95,05 \pm 13,12) \\
\text { e grupo controle }(101,06 \pm 15,04) \text {. } \\
\text { Indivíduos com doença de } \\
\text { Parkinson apresentaram valores } \\
\text { de pico de fluxo expiratório } \\
\text { inferiores em comparação ao } \\
\text { grupo controle com } \mathrm{p}<\mathrm{O}, \mathrm{O} 5 .\end{array}$ \\
\hline
\end{tabular}

CVF: Capacidade Vital Forçada; FMR: Força Muscular Respiratória; PFT: Pico de Fluxo de Tosse; EUA: Estados Unidos da América.

\section{DISCUSSÃO}

A análise da literatura evidenciou que indivíduos com Parkinson apresentam redução da mobilidade torácica, do pico de fluxo de tosse, da força muscular respiratória e de volumes e capacidades pulmonares. Isto pode acontecer em decorrência de uma série de fatores relacionados à sintomatologia clínica motora e/ou não motora apresentada

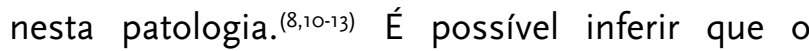
quadro propiciado por esta disfunção tende a gerar distúrbios de ordem estrutural com impacto funcional significativo.

Uma das disfunções encontradas é a redução da mobilidade torácica, avaliada pela cirtometria, que consiste em um conjunto de medidas das circunferências de tórax e abdome durante os movimentos respiratórios. ${ }^{(8,13)}$ Cardoso e Pereira, ${ }^{(8)}$ ao compararem a expansibilidade torácica de não parkinsonianos com a de indivíduos com DP, observaram uma redução estatisticamente significativa neste último grupo. Sugere-se que este quadro seja consequência da sintomatologia típica da DP, pois fatores como a bradicinesia, fixação postural em flexão e a rigidez podem gerar limitações na expansibilidade do tórax e na mobilidade da coluna. ${ }^{(8)}$ A redução da mobilidade torácica pode conduzir a déficits na respiração, impactando na expansibilidade pulmonar e aumentando o trabalho respiratório. ${ }^{(8,13)}$

Outro aspecto relevante da disfunção respiratória na doença de Parkinson é a redução da força muscular respiratória. A avaliação das pressões inspiratória máxima (PImáx) e expiratória máxima (PEmáx) é um método volitivo para aferir a força dos músculos responsáveis por esta função, por meio do manovacuômetro. Observa-se que a PImáx e a PEmáx em indivíduos parkinsonianos encontram-se abaixo do limite da normalidade preconizado para a idade e sexo, havendo maior acometimento na musculatura expiratória, devido 
a maior redução da área de secção transversa dessa musculatura em relação aos músculos inspiratórios. ${ }^{(8,10,11)}$ No estudo de Bonjorni LA et al, ${ }^{(11)}$ constatou-se uma redução estatisticamente significante da PImáx de parkinsonianos quando comparados a idosos hígidos. Esses dados corroboram com outro estudo, onde $68 \%$ dos avaliados apresentaram valores abaixo do limite inferior da normalidade para PImáx e 79\% para PEmáx. ${ }^{(12)}$ Os resultados de uma pesquisa semelhante indicaram que a PImáx e a PEmáx estavam reduzidas em pessoas com DP quando comparadas a um grupo controle. ${ }^{(13)}$ Estes parâmetros se associam negativamente com a gravidade de sintomas respiratórios e a com a progressão da doença. O declínio da força muscular respiratória é justificado pelo aumento da rigidez da caixa torácica, redução de pressão de recolhimento elástico, diminuição do volume de ar e das taxas de fluxo de ar inspirado. ${ }^{(11)}$

Entretanto, Cardoso e Pereira ${ }^{(8)}$ não destacaram diferença significativa entre os valores das pressões inspiratória e expiratórias máximas entre as pessoas com DP e pessoas sem a doença. Estes autores presumiram que a normalidade da Força Muscular Respiratória (FMR) pode ter sido influenciada por fatores como o nível de comprometimento neurológico dos pacientes avaliados em sua amostra.

Distúrbios no mecanismo de tosse contribuem para o surgimento de infecções broncopulmonares que se tornam cada vez mais frequentes com o decorrer da doença, sendo uma importante causa de mortalidade nessa população. Ebihara $S$ et al ${ }^{(14)}$ investigaram a eficácia da tosse em indivíduos com DP nos estágios inicial e avançado comparando-os com um grupo controle. Os grupos em estágio inicial e avançado da doença apresentaram o Pico de Fluxo de Tosse (PFT) menor em comparação ao grupo controle. No entanto, ao comparar-se sujeitos em estágios inicial e avançado não foi observada diferença estatisticamente significante. ${ }^{(14)}$ Resultados de outro estudo indicaram que todos os indivíduos avaliados em seus determinados subgrupos, apresentaram uma tosse ineficaz. ${ }^{(15)}$
Corroborando com estes achados, dados de outra pesquisa demonstraram que $90 \%$ dos parkinsonianos apresentaram valores baixos de pico de fluxo de tosse, tendo relação direta com o alto grau de comprometimento neurológico destes pacientes, com significância estatística. ${ }^{(16)} \mathrm{A}$ redução da força muscular expiratória é um importante fator que contribui para uma tosse ineficaz. ${ }^{(16)}$

Além da diferença da nomenclatura, o pico de fluxo de tosse e o pico de fluxo expiratório são mensurados de forma diferente. No primeiro, geralmente é utilizado um aparelho denominado medidor de pico de fluxo, e a manobra para a avaliação necessita de uma inspiração profunda, fechamento da glote, contração da musculatura expiratória e por final abertura da glote para exalação do fluxo de ar. No pico de fluxo expiratório, a manobra é executada com a glote aberta durante todo o processo que é mensurado com um espirômetro ou medido de pico de fluxo. Apesar das diferenças na execução da manobra, essas duas variáveis tem o mesmo objetivo: avaliar a eficácia do mecanismo de depuração das vias aéreas. ${ }^{(14,15)}$

A espirometria é a medida de ar que entra e sai dos pulmões. A avaliação da função pulmonar por este método constitui-se num importante indicador da condição respiratória de pacientes com DP. ${ }^{\left({ }^{17)}\right.}$ As medidas de Capacidade Vital (CV) e a Capacidade Vital Forçada (CVF), encontram-se abaixo dos valores teóricos preconizados em pacientes parkinsonianos, ${ }^{(8,17,18)}$ e podem ser decorrentes da rigidez da musculatura da caixa torácica e da bradicinesia. ${ }^{(8)}$ Observa-se que ao comparar a função pulmonar de indivíduos com e sem a doença de Parkinson, pessoas com a doença apresentam redução da CV e CVF. ${ }^{(19,20)}$ Entretanto, tais achados podem ter associação com o estágio da doença, visto que Polatli $\mathrm{M}$ et al, (22) encontraram resultados divergentes em indivíduos nos estágios I e II da doença segundo a escala de Hoehn \&Yahr (H\&Y).

Em outro estudo, Shahenn HA et al, (21) investigaram a presença de disfunção pulmonar em DP comparado a um grupo controle e encontraram CVF significativamente reduzida em pessoas com 
DP, e ainda $80 \%$ da população estudada com alteração da função pulmonar, sendo o padrão restritivo o mais comum. As alterações respiratórias restritivas estão associadas a incoordenação do esforço expiratório e diminuição da amplitude torácica, com consequente limitação na elevação das estruturas do tórax e da expansibilidade, além da diminuição da complacência pulmonar. ${ }^{\left({ }^{(8)}\right.}$ Esta incoordenação também foi citada em outra pesquisa, que evidenciou a depleção de neurônios glutamatérgicos centrais responsáveis pelo controle muscular respiratório e sua deterioração em pacientes com DP. ${ }^{(13)}$ Contrapondo tais resultados, um trabalho demonstrou que houve predomínio de distúrbios ventilatórios obstrutivos em toda amostra estudada, o que pode ter relação com o número reduzido da população utilizada. ${ }^{(1)}$ Os distúrbios ventilatórios obstrutivos e restritivos podem estar associados a característica de doenças extrapiramidais, que comumente apresentam incoordenação neuromuscular, afetando significativamente os músculos de vias aéreas superiores e consequente alteração da resistência do fluxo de ar. ${ }^{(13)}$

A terapia medicamentosa tem mostrado influência nos parâmetros de função pulmonar. Embora o uso da Levodopa seja o tratamento medicamentoso mais eficaz disponível para a doença de Parkinson, existem resultados divergentes. ${ }^{(18)}$ Estudos evidenciaram a melhora sobre a função pulmonar após a administração da Levodopa, ${ }^{17,23)}$ com aumento nos valores dos parâmetros avaliados, a exemplo da CVF e do $\mathrm{PFE}^{(23)}$ na fase on da medicação. Credita-se essa melhora à reversão parcial da deficiência motora, resultando em diminuição do tremor e rigidez muscular, melhora na coordenação dos músculos e facilitação do movimento. ${ }^{(19)}$ Guedes, ${ }^{(24)}$ ao investigarem a PImáx e a PEmáx no período on e off da medicação, comparando a um grupo controle, encontraram valores menores em ambos os períodos, estando essa disfunção respiratória presente independente do estadiamento da doença. Os resultados de outra pesquisa indicaram que apenas indivíduos que apresentavam obstrução das vias aéreas superiores obtiveram resultados significativos após a utilização da levodopa. ${ }^{(25)}$ A piora dos resultados na fase on da medicação para a variável CVF, sem modificação entre os períodos para os valores de PFE foi evidenciada em um estudo, sendo justificada pelos próprios autores como falhas não identificadas na metodologia. ${ }^{(18)}$

As disfunções respiratórias ocorridas em indivíduos com doença de Parkinson podem ser identificadas através de métodos acessíveis e de baixo custo. $A$ análise da função pulmonar permite identificar precocemente alterações que possam impactar diretamente na funcionalidade desses pacientes, propiciando o direcionamento das ações e aprimorando as estratégias de assistência ao indivíduo com doença de Parkinson.

\section{CONSIDERAÇÕES FINAIS}

O presente estudo sugere que pessoas com doença de Parkinson apresentam redução da mobilidade torácica, do pico de fluxo de tosse, da força muscular respiratória e de volumes e capacidades pulmonares, o que pode levar ao comprometimento da funcionalidade destes indivíduos. A falta de uniformidade nos métodos de avaliação da função pulmonar nos estudos dificultou a caracterização e comparação dos desfechos. Salienta-se que a avaliação da função pulmonar é essencial para o diagnóstico, intervenção e acompanhamento de pessoas com condições de saúde com repercussões respiratórias, como ocorre na DP.

\section{INFORMAÇÕES ADICIONAIS}

who Grupo de Pesquisa Estudo e Pesquisa da Funcionalidade, em adição aos autores principais: Adriana Borges Leite, ${ }^{a}$ Isabela Barboza Gomes, ${ }^{\text {b }}$ Luis Gustavo de Souza Carvalhoc e Nicole Nunes Souza Costa. ${ }^{d}$

*⿰丬火火火 Grupo de Pesquisa DINEP - Divisão de Neurologia e Epidemiologia/UFBA, em adição aos autores principais: Caroline Ferreira Guerreiro. ${ }^{\mathrm{e}}$ 
a) Fisioterapeuta, Professora Assistente, Universidade Católica do Salvador

b) Isabela Barboza Gomes - Fisioterapeuta, Hospital Santa Izabel, Salvador, Bahia.

c) Luis Gustavo de Souza Carvalho - Fisioterapeuta, Departamento de Ciências da Vida, Universidade do Estado da Bahia.

d) Nicole Nunes Souza Costa - Fisioterapeuta, Departamento de Ciências da Vida, Universidade do Estado da Bahia.

e) Fisioterapeuta do Serviço de Fisioterapia do Hospital Geral Roberto Santos

\section{REFERÊNCIAS}

1. Souza CFM, Almeida HCP, Sousa JB, Costa $\mathrm{PH}$, Silveira YSS, Bezerra JCL. A Doença de Parkinson e o processo de envelhecimento motor: uma revisão de literatura. Rev Neurocienc. 2011;19(4):718-723.

2. Kalia LV, Lang AE. Parkinson's disease. Lancet. 2015;386: 896-912.

3. Díaz F, Mena I, Gómez A. Diagnóstico precoz de enfermedad de Parkinson: neurospect del transportador de Dopamina. Rev Chil NeuroPsiquiat 2002;40:243-52. Available from: http://www.scielo.cl/scielo.php?script=sci_arttext \&pid=S0717-92272002000300006

4. Bellucci A, Mercuri NB, Venneri A, Faustini G, Longhena F, Pizzi M, et al. Review: Parkinson's disease: from synaptic loss to connectome dysfunction. Neuropathol Appl Neurobiol. 2016; 42(1):77-94. Available from: http://www.ncbi.nlm. nih.gov/pubmed/26613567

5. Sant CR, Oliveira SG, Rosa EL, Sandri J, Duarte $M$, Posser SR. Abordagem fisioterapêutica na Doença de Parkinson. RBCEH. [Internet]. 2008;5(1):80-89. Available from: http://www. upf.br/seer/index.php/rbceh/article/view/259

6. Reijnders JSAM, Ehrt U, Webwe WEJ, Aarsland $D$, Leentiens AFG. A systematic review of prevalence studies of depression in Parkinson's disease. Movement Disorders. [Internet]. 2008;23(2):183-189. Available from: http://www. ncbi.nlm.nih.gov/pubmed/17987654
7. Vercueil L, Linard JP, Wuvam B, Pollak P, Benchetrit $G$. Breathing pattern in patients with Parkinson's disease. J Respir Physiol. [Internet]. 1999;118(2-3):163-172. Available from: http:// www.sciencedirect.com/science/article/pii/ SO034568799000754

8. Cardoso SRX, Pereira JS. Análise da função respiratória na doença de Parkinson. Arquivos de Neuropsiquiatria. [Internet]. 2002; 60(1): 9195. Disponível em: http://www.scielo.br/pdf/anp/ v60nl/8237.pdf

9. Alves AL, Coelho AC, Brunetto, AF. Fisioterapia respiratória na doença de Parkinson idiopática: relato de caso. Fisioter Pesqui. [Internet]. 2005;12(3):46-49. Disponível em: http://www.revistas.usp.br/fpusp/article/ download/76717/80540

1O. Kim J, Davenport P, Sapienza C. Effect of expiratory muscle strength on elderly cought function. Arch Gerontol Geriatr. 2009; 48361 66. Available from: http://www.ncbi.nlm.nih.gov/ pubmed/18457885

11. Bonjorni LA, Jamami M, Lorenzo VAP, Pessoa VP. Influência da doença de Parkinson em capacidade física, função pulmonar e índice de massa magra corporal. Fisioter Mov. [Internet]. 2012;25(4):727-736. Available from: http://www. scielo.br/pdf/fm/v25n4/aO5v25n4.pdf

12. Seccombe LM, Giddings HL, Rogers PG, Corbett AJ, Hayes MW, Peters MJ, Veitch EM. Abnormal ventilatory control in Parkinson's diseaseFurther evidence for non-motor dysfunction. Respir Physiol Neurobiol. [Internet]. 2011;179(23):300-304. Available from: http://www.ncbi. nlm.nih.gov/pubmed/21982817

13. Wang Y, Shao W-b, Gao L, Lu J, Gu H, et al. Abnormal Pulmonary Function and Respiratory Muscle Strength Findings in Chinese Patients with Parkinson's Disease and Multiple System Atrophy-Comparison with Normal Elderly. PLoS ONE. [Internet]. 2014; 29;9(12). Available from: http://www.ncbi.nlm.nih.gov/pubmed/25546308

14. Ebihara S, Saito H, Kanda A, Nakajoh M, Takahashi H, Sasaki H. Impaired Efficacy of Cough in Patients With Parkinson Disease. CHEST. [Internet]. 2003;124(3):1009-1915. Available from: http://www.ncbi.nlm.nih.gov/ pubmed/12970031 
15. Silverman EP, Carnaby G, Singletary F, Ruddy BH, Yeager J, Sapienza C. Measurement of Voluntary Cough Production and Airway Protection in Parkinson Disease. Archives of Physical Medicine and Rehabilitation. [Internet]. 2016;97(3):413-2O. Available from: http://www. ncbi.nlm.nih.gov/pubmed/26551228

16. Ramos ML, Neves DR, Lima VP, Orsini M, Machado D, Bastos VHV, et al. Análises de parâmetros pneumofuncionais em pacientes com doença de Parkinson: estudo piloto. Revista Brasileira de Neurologia. [Internet]. 2014;5O(2):38-43. Available from: http://files. bvs.br/upload/S/O1O1-8469/2O14/v5On2/ a4215.pdf

17. Pandis MF, Starace A, Stefanelli F, Marruzzo P, Meoli L, De Simone G. Modification of respiratory function parameters in patients with severe Parkinson's disease. Neurol Sci. [Internet]. 2002;23(2):69-70. Available from: http://www.ncbi.nlm.nih.gov/pubmed/12548348

18. Lim A, Leow L, Huckabee ML, Frampton C, Anderson T. A Pilot Study of Respiration and Swallowing Integration in Parkinson's disease: "On" and "Off" Levodopa. Dysphagia. [Internet]. 2008;23(1):76-81. Available from: http://www. ncbi.nlm.nih.gov/pubmed/17602261

19. Sathyaprabha TN, Kapavarapu PK, Pal PK, Thennarasu K, Raju TR. Pulmonary Functions in Parkinson's disease. Indian J Chest Allied Sci. [Internet]. 2005; 47(4): 251-257. Available from: http://www.ncbi.nlm.nih.gov/pubmed/16255396.
2O. Owolabi LF, Nagoda M, Babashani M. [Internet]. 2016;19(1):66-70. Available from: http://www.ncbi.nlm.nih.gov/pubmed/26755221

21. Shahenn HA, Ali MA, Elzaher MAA. Parkinson's Disease and Pulmonary Dysfunction [Internet]. Egypt J Neurol Psychiat Neurosurg. 2009;46(1):129-140. Available from: https:// www.researchgate.net/publication/266872230_ Parkinson's_Disease_and_Pulmonary_Dysfunction.

22. Polatli M, Akvol A, Cildag O, Bayulkem K. Pulmonary function tests in Parkinson's disease. European Journal of Neurology. [Internet]. 2001;8(4):341-345. Available from: http://www. ncbi.nlm.nih.gov/pubmed/11422431

23. Pal PK, Sathyaprabha TN, Tuhina P, Thennasrasu K. Pattern of Subclinical Pulmonary Dysfunctions in Parkinson's disease and the Effect of Levodopa. Movement Disorders. [Internet]. 2007;22(3):420-424. Available from: http://www.ncbi.nlm.nih.gov/ pubmed/17230476

24. Guedes LU, Rodrigues JM, Fernandes AA, Cardoso FE, Parreira VF. Respiratory Changes in Parkinson Disease May be Unrelated to Dopaminergic Dysfunction. Arq Neuropsiquiatr. [Internet]. 2O12;7O(11):847-851. Available from: http://www.ncbi.nlm.nih.gov/pubmed/23175196

25. Herer B, Arnulf I, Housset B. Effects of Levodopa on Pulmonary Function in Parkinson's disease. CHEST. [Internet]. 2001;119(2):387-393. Available from: http://www.ncbi.nlm.nih.gov/ pubmed/11171713 